\title{
The effects of uniform field flicker and blurring on the global precedence effect
}

\author{
WILLIAM J. LOVEGROVE \\ University of Wollongong, Wollongong, New South Wales, Australia \\ and \\ STEPHEN LEHMKUHLE, JOHN A. BARO, and RALPH GARZIA \\ University of Missouri, St. Louis, Missouri
}

\begin{abstract}
The involvement of low-level visual processes in the global precedence effect was investigated by selectively manipulating the low and high spatial frequency contribution by uniform field flicker masking and blurring, respectively. Uniform field flicker increased reaction times for the global stimuli and decreased reaction times for local stimuli. Blurring had little effect on reaction times for global stimuli but increased reaction times for local stimuli. The results support the proposal that the global precedence effect is partially a result of low-level visual mechanisms.
\end{abstract}

In the human visual system, the rate of temporal processing decreases with increasing spatial frequency. This spatiotemporal interaction has been demonstrated with reaction time (Breitmeyer, 1975), visible persistence (Meyer \& Maguire, 1977), and cortical evoked potentials (Parker \& Salzen, 1977). These spatiotemporal interactions seem to reflect a global-to-local mode of visual information processing. The global, coarse configurational properties of the spatial stimulus are processed by fastacting lower spatial frequency mechanisms, and the local spatial details are subsequently processed by slower acting higher spatial frequency mechanisms.

It is possible that the spatiotemporal properties of spatial frequency channels are important in higher order perceptual tasks, such as that devised by Navon (1977). In Navon's task, subjects are shown large letters composed of smaller letters. The small and large letters may be consistent or inconsistent with each other. Navon found that subjects are significantly faster in responding to the global as opposed to the local letters (the global precedence effect). Furthermore, subjects respond more slowly to the inconsistent stimuli as opposed to the consistent stimuli in the local but not the global condition.

A number of researchers have demonstrated that postperceptual processes (e.g., attention or response competition) influence the magnitude of the global precedence effect (Boer \& Keuss, 1982; Garner, 1983; Miller, 1981; Shulman, Sullivan, Gish, \& Sakoda, 1986).

If the low-level visual processes that contribute to the global precedence effect are the same as the processes that are revealed in psychophysical and physiological investigations of the influence of spatial frequency on reaction

Correspondence should be addressed to William J. Lovegrove, Department of Psychology, University of Wollongong, Wollongong, New South Wales 2500, Australia. time, visible persistence, and evoked potentials, the global precedence effect should be influenced by manipulations that are known to influence those phenomena. For instance, it has been shown that a uniform field flicker (UFF) mask increases reaction times, evoked potential latencies, as well as contrast detection thresholds for low but not high spatial frequencies (Baro \& Lehmkuhle, 1989, 1990; Breitmeyer, Levi, \& Harwerth, 1981). To the extent that the global precedence effect also reflects the differential processing of low and high spatial frequencies, its magnitude should be reduced in the presence of a UFF mask. Similarly, blurring, which primarily reduces visibility of high and not low spatial frequencies, should increase the magnitude of the global precedence effect. These effects of UFF and blur were investigated here.

\section{METHOD}

\section{Subjects}

Ten subjects (including the authors) with normal or corrected-to-normal visual acuity participated in each experiment.

\section{Stimuli}

The stimuli consisted of the global characters $\mathrm{H}$ and $\mathrm{S}$, which were composed of $6 \times 7$ character arrays of the local letter $H$ or $S$, thus resulting in four possible stimulus combinations. The sizes of the global and local characters were $2.15^{\circ} \times 1.43^{\circ}$ and $0.29^{\circ} \times 0.19^{\circ}$, respectively, at the viewing distance of $120 \mathrm{~cm}$. The stimuli were presented on a standard Apple II monitor, which was covered with a white paper mask. A circular hole was cut in the center of the mask (diameter, $3.34^{\circ}$ ), through which the stimulus could be seen. A uniform flickering field background was created by a strobe light (STROBOSLAVE, Type 1539-

A) set at high intensity, positioned to the side of the observer, and pointed at the monitor screen. The strobe light flickered on and off at a rate of $12 \mathrm{~Hz}$.

In the unblurred conditions, the stimulus area of the screen was uncovered, whereas in the blurred conditions, it was covered with a diffusing screen. In order to characterize the spatial transfer properties of the diffuser, contrast detection thresholds were obtained for sine-wave grating patterns conventionally generated on a CRT display (Tektronix 608, P31 phosphor). The diffuser substantially reduced the contrast of high spa- 
tial frequency components of the stimuli (i.e., above $3 \mathrm{cpd}$ ), but it had a much smaller effect on the contrast of lower spatial frequency components (i.e., $3 \mathrm{cpd}$ and below).

The intensity and contrast of the monitor were adjusted so that when each pixel in a small region of the screen was illuminated, the luminance was $53.7 \mathrm{~cd} / \mathrm{m}^{2}$. When no pixels were illuminated, the luminance was $0.2 \mathrm{~cd} / \mathrm{m}^{2}$. With the diffusing screen covering the monitor, the luminance was reduced by $18 \%$.

\section{Procedure}

Each trial consisted of the following sequence. A warning tone followed by a variable interval (randomly selected from 100 to $500 \mathrm{msec}$ ) preceded the stimulus. The subjects were instructed to indicate the display letter as quickly and as accurately as possible. The subjects responded by pressing one of two buttons on a joystick to indicate the letter $\mathrm{H}$ or $\mathrm{S}$.

Within a trial block, each subject was instructed to attend to either local or global letters. A trial block consisted of 80 trials, 20 for each of the four possible combinations of $\mathrm{Hs}$ and Ss, presented in a random order. Each subject participated in four trial blocks. In each of the steady and the UFF conditions, one block of trials was run with attention directed to the global features (composite letters), and another block was run with attention directed to the local features (component letters).

The steady and the UFF conditions were blocked, and the sequence alternated between subjects. The global and local trials were also ordered alternately. In the blurred conditions, the same procedures were utilized, except that the diffusing screen was placed over the monitor.

\section{RESULTS}

The mean reaction time (RT) of the correct responses for each condition was calculated for each subject. Average RTs across observers for each condition are displayed in Figure 1. Accuracy levels were comparable in all conditions, and RTs for incorrect responses were not analyzed.

\section{Unblurred}

In Figure 1A, it can be seen that in all stimulus conditions, mean RT was faster when the subject's attention was directed to global as opposed to local features $[F(1,9)$ $=13.20, p<.006]$. The range of the global precedence effect varied from $75 \mathrm{msec}$ in the steady background condition to around $40 \mathrm{msec}$ in the UFF conditions. The flickering background therefore reduced the magnitude of the global precedence effect. The presence of UFF increased global RT by about $25 \mathrm{msec}[t(19)=4.48, p<.001]$, and it decreased local RT by $10-30 \mathrm{msec}[t(19)=2.41$, $p<.03]$. Accordingly, there was no main effect of background on RT $[F(1,9)<1]$, but there was a significant interaction effect [i.e., global-local $\times$ background; $F(1,9)$ $=11.94, p<.008]$. There was a main effect of letter consistency $[F(1,9)=64.15, p<.001]$; however, there was no interaction effect between letter consistency and global-local $[F(1,9)<1]$.

\section{Blurred}

The data for the blurred conditions are shown in Figure 1B. With blur, the mean RT was again faster when the subject's attention was directed to global as opposed to local features $[F(1,9)=57.20, p<.001]$. The range of the global precedence effect in the presence of blur varied from about $75 \mathrm{msec}$ with the UFF background to
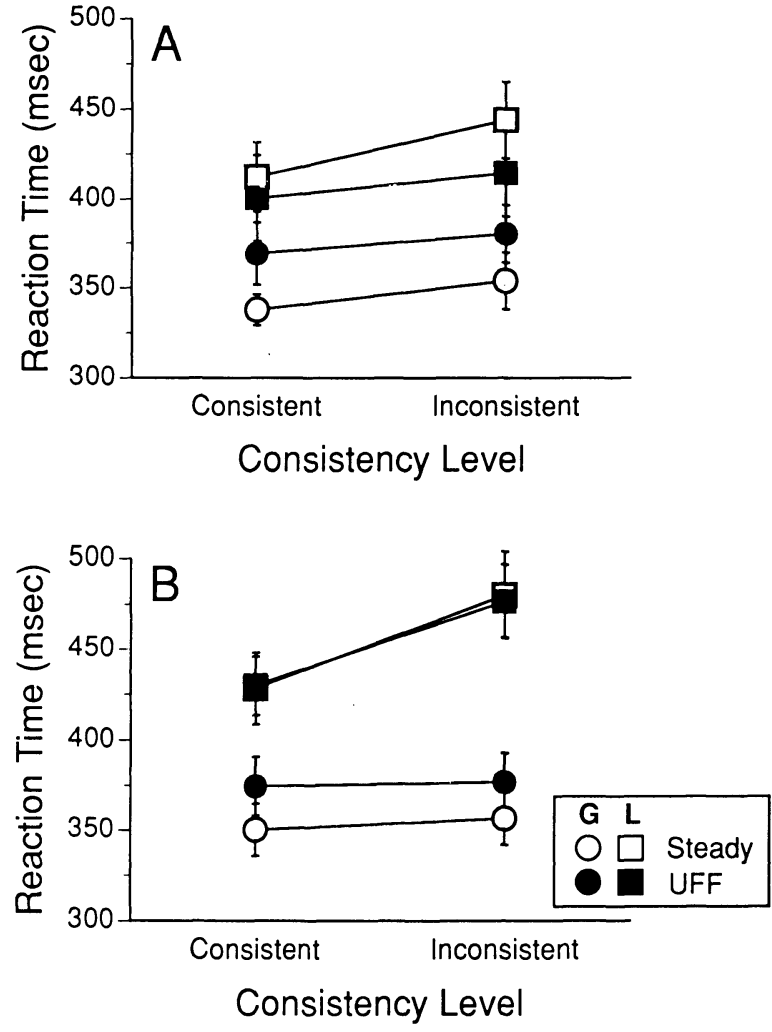

Figure 1. Reaction times to the global (G) and local (L) stimuli in all conditions. Figure $1 \mathrm{~A}$ shows the results for consistent and inconsistent stimuli under steady viewing conditions (open symbols) and in the presence of uniform field flicker (UFF; filled symbols) in the unblurred condition. Figure 1B shows the blurred condition; conventions are the same.

about 100 msec with the steady background. Overall, there were marginal effects of background $[F(1,9)=3.91$, $p<.08]$ and of interaction between background and global-local $[F(1,9)=4.71, p<.06]$. In contrast to the unblurred condition, the only effect of introducing flicker to the background was to increase global RTs $20-25 \mathrm{msec}$ $[t(19)=5.15, p<.001]$; there was no effect of background on local RT $[t(19)=0.16$, n.s.]. There was also a main effect of letter consistency $[F(1,9)=50.14$, $p<.001$, and an interaction between letter consistency and global-local $[F(1,9)=27.31, p<.001]$.

\section{DISCUSSION}

In the UFF conditions, the flickering background increased RTs to the global stimuli while it decreased RTs to the local stimuli. The former result is consistent with the concept that the global precedence effect, under the present stimulus conditions, is mediated by the fast, low spatial frequency mechanisms that are adapted by the UFF mask.

The decrease in RT to the local stimuli was not expected and is of theoretical interest. It is consistent with a number of previous studies that have shown facilitatory effects of UFF at high spatial frequencies with a range of measures (Badcock \& Smith, 1989; Bowling, 1985). These results, together with the present observation of decreased RTs to local stimuli, are consistent with the idea that slower, high spatial frequency mechanisms are disinhibited when the fast, low spatial frequency mechanisms are masked by the UFF. Regardless of the reasons 
for the facilitation of local information, the fact that it was observed indicates that the increase in global RT did not result from a general decrease in visibility of the stimuli with UFF.

These results of UFF are similar to previous studies in which sinewave gratings have been used as stimuli. Furthermore, it is important in terms of previous reports which suggest that the global precedence effect was primarily postperceptual (Boer \& Keuss, 1982; Garner, 1983; Miller, 1981; Shulman et al., 1986).

The main effect of blurring was to slow the RTs for the local stimuli in both conditions, with the effect being greater for the UFF than for the steady condition. The effect of UFF flicker on local RTs was counteracted by the effect of blurring.

The combined effect of these two manipulations indicates that one of the important factors in the global precedence effect is the relative timing of the transmission of low and high spatial frequency information. The magnitude of the effect can be selectively altered by manipulating the rate of transmission of either the low or the high spatial frequencies.

In conclusion, the results reported here demonstrate the involvement of low-level visual mechanisms in the global precedence effect without excluding the possibility that higher level processes also make some additional contribution.

\section{REFERENCES}

BADCOCK, D., \& SMITH, D. (1989). Uniform field flicker: Masking and facilitation. Vision Research, 29, 803-808.

Baro, J. A., \& LehmKuhle, S. (1989). The effects of a luminancemodulated background on grating-evoked cortical potentials in the cat. Visual Neuroscience, 3, 563-572.

Baro, J. A., \& LehmKuhle, S. (1990). The effects of a luminancemodulated background on human grating-evoked cortical potentials. Clinical Vision Science, 5, 265-270.
Boer, L. C., \& Keuss, P. J. G. (1982). Global precedence as a postperceptual effect: An analysis of speed-accuracy tradeoff functions. Perception \& Psychophysics, 31, 358-366.

Bowling, A. (1985). The effects of peripheral movement and flicker on the detection thresholds of sinusoidal gratings. Perception \& Psychophysics, 37, 181-188.

BREITMEYER, B. (1975). Simple reaction time as a measure of the temporal response properties of transient and sustained channels. Vision Research, 15, 1411-1412.

BreitMEYer, B., LeVI, D., \& HARWERTH, R. (1981). Flicker masking in spatial vision. Vision Research, 21, 1377-1385.

GARNER, W. (1983). Asymmetric interactions of stimulus dimensions in perceptual information processing. In T. Tighe \& B. Shepp (Eds.), Perception, cognition and development: Interactional Analyses (pp. 138). Hillsdale, NJ: Erlbaum.

MEYer, G., \& MAGUIRE, W. (1977). Spatial frequency and the mediation of short-term visual storage. Science, 198, 524-525.

Miller, J. (1981). Global precedence in attention and decision. Journal of Experimental Psychology: Human Perception \& Performance, 7, 1161-1174.

Navon, D. (1977). Forest before trees: The precedence of global features in visual perception. Cognitive Psychology, 9, 353-383.

PARKER, D., \& SALZEN, E. (1977). Latency changes in the human visual evoked response to sinusoidal gratings. Vision Research, 17, 1201-1204.

Shulman, G., Suluivan, M., Gish, K., \& Sakoda, J. (1986). the role of spatial frequency channels in the perception of local and global structure. Perception, 15, 259-273.

(Manuscript received December 3, 1990.) 